\title{
STEMI Networks in the COVID-19 Era
}

\author{
Theodora Benedek ${ }^{1,2}$ \\ ${ }^{1}$ Clinic of Cardiology, Emergency Clinical County Hospital, Târgu Mureș, Romania \\ 2 "George Emil Palade" University of Medicine, Pharmacy, Sciences and Technology, Târgu Mureș, Romania
}

The COVID-19 pandemic impacts the management of cardiovascular emergencies in one of the most dramatic ways encountered in the history of modern cardiology.

The relationship between SARS-CoV-2 infection and cardiovascular emergencies is bi-directional. From a logistic point of view, the management of cardiovascular emergencies is significantly affected by the COVID-19 pandemic. At the same time, the most severe forms of viral infection are encountered in patients with cardiovascular diseases (CVD).

A recent survey conducted by the European Society of Cardiology (ESC) showed a dramatic decrease in the number of primary percutaneous interventions (pPCI) performed in patients with ST-segment elevation acute myocardial infarction (STEMI) across Europe. ${ }^{1}$ This was mainly caused by the fear of patients to present to STEMI hospitals or to call 112 when they had a chest pain, because they were more scared by the possibility to contact the new coronavirus than by the possibility to have a heart attack.

This survey indicates that more than $80 \%$ of European centers are encountering a significant reduction in the number of pPCIs performed during the COVID-19 pandemic, most of them reporting decreases between 50\% and 70\% in the number of $\mathrm{pPCI}$ procedures. At the same time, more than $50 \%$ of pPCI centers reported that patients arrive later than usual for pPCI, beyond the optimal time window for revascularization.1 New logistic challenges include the need to exclude Sars-CoV2 infection in patients presenting to the emergency room and the need to take appropriate measures to protect medical staff in the emergency room and cath lab. These all take extra time, while we have learned very well in the latest years that time is muscle, time is life.
As a consequence, in a recent material released by the ESC to serve as guidance for diagnosis and management of CVD during the COVID-19 pandemic, the ESC recommends to calculate an extra time of 60 minutes in the original time frame of maximum 2 hours from diagnosis to pPCI in the guidelines. ${ }^{2}$ Pragmatically speaking, this means that we have only one hour left, instead of two, to transport the patient to the closest cath lab, because we will lose one hour with extra logistic measures specific for the COVID-19 period. This may significantly impact the choice of pPCI versus thrombolysis, leading to an increased number of treatment options in favor of thrombolysis.

A recent correspondence from this year compared the emergency admissions for acute coronary syndromes (ACS) in 15 hospitals from Northern Italy in February-March 2020 versus January-February 2020 and the same period in 2019, and reported a significant decrease in the total number of ACS admissions in the COVID-19 period: 13.3 daily admissions for ACS vs. 18 daily admissions in the preceding month ( $\mathrm{p}<0.001$ ) and vs. 18.9 in the same period of 2019 (p $<0.001){ }^{3}$ Another study analyzing ACS admissions in Austria reported a $39.4 \%$ decrease in the number of ACS admissions (25.53\% for STEMI and $49.24 \%$ for NSTEMI) as a result of the COVID-19 pandemic. ${ }^{4}$ As for the network times, a study from Hong Kong reported a significant prolongation of critical times in STEMI networks: 318 minutes in the COVID period vs. 82.5 minutes in the pre-COVID months for the time from symptoms onset to presentation, and 110 minutes vs. 84.5 minutes for door-to-balloon time. ${ }^{5}$

Unpublished data from Romanian centers indicate a dramatic decrease of $75 \%$ in the number of pPCI procedures for STEMI compared to the pre-COVID period, while 
the number of patients referred from territorial hospitals to STEMI hubs decreased by almost 70\%.

All these data demonstrate a very strong impact of the COVID-19 pandemic on the functionality of STEMI networks and raise serious concerns related to the long-term effects of this devastating disease on patients with CVD.

\section{CONFLICT OF INTEREST}

Nothing to declare.

\section{REFERENCES}

1. STEMI admissions during COVID-19. Available at: https:// www.escardio.org/Education/COVID-19-and-Cardiology/ esc-survey-on-stemi-admissions-during-covid-19
2. ESC Guidance for the Diagnosis and Management of CV Disease during the COVID-19 Pandemic. Available at: https:// www.escardio.org/Education/COVID-19-and-Cardiology/ ESC-COVID-19-Guidance

3. De Filippo O, D'Ascenzo F, Angelini F, et al. Reduced Rate of Hospital Admissions for ACS during Covid-19 Outbreak in Northern Italy [published online ahead of print, 2020 Apr 28]. N Engl J Med. 2020;NEJMc2009166. doi: 10.1056/ NEJMc2009166.

4. Metzler B, Siostrzonek P, Binder RK, Bauer A, Reinstadler SJ. Decline of acute coronary syndrome admissions in Austria since the outbreak of COVID-19: the pandemic response causes cardiac collateral damage. Eur Heart J. 2020;41:18521853. doi:10.1093/eurheartj/ehaa314

5. Tam CF, Cheung KS, Lam S, et al. Impact of Coronavirus Disease 2019 (COVID-19) Outbreak on ST-Segment-Elevation Myocardial Infarction Care in Hong Kong, China. Circ Cardiovasc Qual Outcomes. 2020;13:e006631. doi: 10.1161/ CIRCOUTCOMES.120.006631. 